\title{
Pulmonary Embolus after a Dental Visit
}

\author{
M. Sami Valid, MD,
}

Locum Tenens, Wisconsin

Pulmonary embolism (PE) is one of the most common cause of cardiovascular death in the country. As notorious as stroke in the community PE is one of the most dreaded causes of death in hospitalized people who must remain in bed for a long time. Blood clots develop in lower extremities because of sluggish blood flow, especially in the deep veins of legs, called deep venous thrombosis (DVT). When a piece of a clot in a deep vein, often in a leg, breaks off and travels to the lungs, passing through the right atrium and ventricle into the pulmonary arteries and causing pulmonary embolism.

Vein blood clots occur more often in people who are not very mobile. The extreme example is someone who's had a stroke and is paralyzed. The risk of having a blood clot there is one in two. However, the possible of developing pulmonary embolism in a healthy lady without prior history of stroke, thrombophilia or known risk factors such as long flight or travel is not totally nil.

In my previous paper I presented a case of right atrial embolism with fatal outcome. In this paper I present a case of pulmonary embolism that is fortunately diagnosed and treated with favorable outcome so far and which has unusual triggering circumstances.

The patient is a 75 year-old practically healthy lady, not on a lot of medications (calcium supplement, vitamins and hemorrhoid topical hydrocortisone) presented with complaints of chest tightness for several days associated with right calf discomfort. The patient reports she had tooth extraction 5 days before and was spent 4 hours in chair. She denies history of blood clots and she is not on systemic hormones nor a smoker. She never had heart surgery or abdominal surgery. She has history of cystocele and proctocele and had vaginal hysterectomy \& oophorectomy the year before. She denied fever, chills, abdominal pain or change in stool or urine. Patient did not appear to bein distress and her saturation is in upper 90s on NC. Electrocardiogram shows normal sinus rhythm, troponin normal and chest x-ray unremarkable. Chest CT angiography showed bilateral lower lobe pulmonary emboli, thrombus burden greater on the right. Patient was started on therapeutic enoxaparin for pulmonary embolism/deep venous thrombosis. The caveats of anticoagulation and risk of hemorrhoidal bleeding explained to the patient.

This is a case of PE triggered by a visit to the dentist; it is rare but can happen $[1,2]$. The case demonstrates that a patient with no remarkable comorbidities can easily develop DVT and later PE in the few days following an outpatient procedure requiring sitting for several hours. This case may provide anecdotal basis for the rationale to administer a subcutaneous dose of heparin for DVT/PE risk reduction purposes before long and probably short dental procedures. We need always to remember that approximately 5-8\% [2] of the U.S. population have genetic risk factors for inherited thrombophilias that increases the risk for unexpected thrombosis. Furthermore, PE is often undiagnosed and the true death rate is almost certainly substantially higher that reported.

\section{REFERENCES}

[1] Garfunkel A, Galili D, Findler M, Zusman SP, Malamed SF, Elad S, Kaufman E. Chest pains in the dental environment. Refuat Hapeh Vehashinayim (1993). 2002 Jan;19(1):51-9, 101.

[2] Freeman NS, King RA, Plezia RA, Shearer HT. Office emergencies: an outline of causes, symptoms, and treatment. J Am Dent Assoc. 1977 Jan;94(1):91-6.

[3] Venous thromboembolism (blood clots). Data \& Statistics. CDC. http://www.cdc.gov/ ncbddd/dvt/data.html 